\title{
Human-Centered Intelligent Systems
}

\author{
Rajiv KHOSLA*
}

\begin{abstract}
The transition of intelligent systems from laboratories to real world in the last decade has created a need for humanization of intelligent systems. In this paper we outline human-centered framework for development of intelligent systems. A distributed multi-layered multi-agent architecture is one of the four components of the framework. We describe in this paper that how the multi-layered architecture facilitates human-centered modelling of intelligent systems from multiple perspectives. The framework and the distributed multi-layered multi-agent ontology has been applied in a range areas including alarm processing, web mining, image processing, sales recruitment and medical diagnosis.
\end{abstract}

Keywords: Human-centeredness, soft computing, intelligent agents, multiple perspectives

\section{Introduction}

Intelligent systems today are being applied in a range of areas including image processing, engineering, process control, data mining, internet and others. In the process of applying intelligent systems to complex real world problems three phenomena have emerged. Firstly, the application of intelligent agents in distributed environments has resulted in merger of techniques from soft computing area with those in distributed artificial intelligence. Secondly, in an effort to improve the quality of solution of intelligent systems employing soft computing paradigms (like fuzzy logic, neural networks, and genetic algorithms), researchers have been combining and fusing technologies. This has resulted in hybrid configurations of soft computing technologies. Finally, from a practitioners perspective, as the soft computing technologies have moved out of laboratories into the real world the need for practitioner-centered technology independent constructs for modeling problems involving soft computing technologies has emerged. In this paper we describe a distributed multi-layered multi-agent soft computing architecture which models these three phenomena. The multi-agent soft computing architecture is one of the four components of a humancentered intelligent system development framework. We show with the help of examples how the

* School of Business, La Trobe University, Melbourne Victoria-3086, Australia multi-agent architecture facilitates human-centered modelling of intelligent systems. The humancentered system development framework is motivated by the human-centred approach and criteria outlined in the $1997 \mathrm{NSF}$ workshop on human-centered systems and consistent problem solving structures/strategies employed by practitioners while designing solutions to complex problems or situations.

The paper is organised as follows. Section 2 describes human-centered approach and the criteria for developing human-centered intelligent. Section 3 outlines the four components of the human-centered system development framework and how these criteria relate to human-centered research and design criteria described in section 2. Section 4 describes the design of distributed multi-layered multi-agent architecture describes Section 5 describes with help of examples how the various layers of the architecture facilitate human-centered modeling from multiple perspectives. Section 6 concludes the paper.

\section{Human-Centered Approach}

Human-centered development is about achieving synergy between the human and the machine. This synergism goes (as outlined in the preceding section) beyond human-computer interaction concepts, people in the loop philosophy and other interpretations given to human-centeredness. Although most systems are designed with some consideration of its human users, most are far from human-centered. 
The informal theme of the recently held NSF workshop on human-centered systems (1997) was people propose, science studies, and technology conforms. In other words, humans are the centerpiece of human-centered research and design (as shown in Figure 1). They are the prime drivers and technology is a primitive that is used -based on its conformity to the needs of people in a field of practice. The three criteria laid down in the workshop for human -centered system development are:

1. Human-Centered research and design is problem/need driven as against abstraction driven (although there is an overlap)

2. Human-Centered research and design is activity centered

3. Human-Centered research design is context bound.

The first criteria outlines a need for developing software systems that are modeled based on how people use various artifacts to solve problems in a field of practice. In other words, technology independent constructs need to be defined which model information processing mechanisms and models of people in a field of practice. These constructs should also facilitate modeling of problem solving context which may involve range of situations both normal as well as exceptional. For example, a power system control operator, acknowledges only a limited number of alarms when the power system network is in a steady state. However, when power system network is in an emergency or unsta-

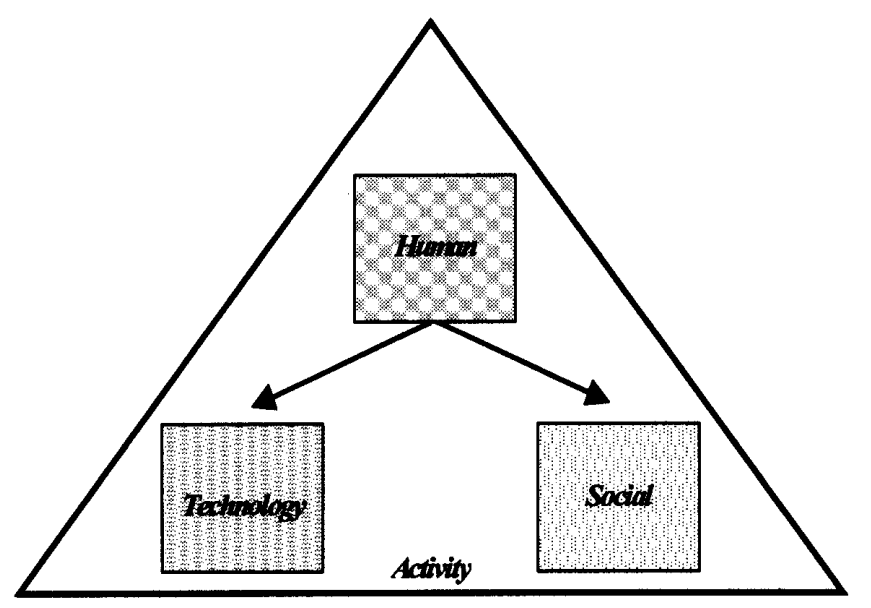

Figure 1: A Human-Centered Approach Leading to Successful Systems ble state (e.g., blackout) hundreds of alarms are displayed on the monitor and the operator has to diagnose the fault in a limited time frame. The decision making paths adopted by the operator are different in these two contexts.

Further, this criteria also suggests that abstract theories like graph theory or Allogic or other domain theories are not necessarily applicable or intuitive in a problem driven environment although, consistent problem solving structures employed by people in various fields of practice may be employed.

The second criterion emphasizes system development based on practitioners or users goals and tasks rather than system designer's goals and tasks. In other words, this criteria emphasizes the need for situating the problem in the user's work environment for modeling user's goals and tasks.

The technologies used to accomplish the tasks should be able to satisfy the task constraints as well as optimize the quality of solution and performance of the system. For example, during an emergency state in a power system control centre, the operator has a limited time for processing the alarms and diagnosing the fault in the power system network. Thus a parallel processing soft computing technique like artificial neural network can be used to reduce the search time. Further, given the uncertain and nondeterministic nature of complex real world problems and corresponding imprecise and approximate solutions provided by soft computing technologies it is important to optimize the quality of solution as well the performance of the technologies. The optimization process can occur in two stages. The first stage may involve hybridisation of various technologies (to capture their individual strengths). The second stage may involve direct human feed-

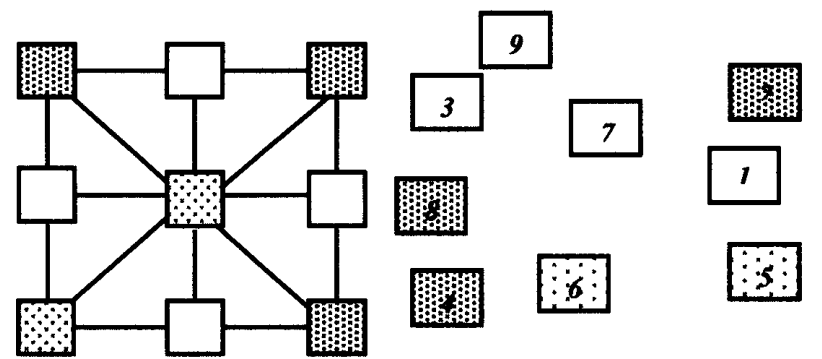

(a) Task(Perceptual): Identify (b)Task(Perceptual \&Cognitive): circles, Search three circles with Color three circles whose same color on a straight line numbers add to 15

Figure 2: Two Representations of Tic-Tac-Toe 
back to the system for improving quality of solution provided by the technologies used.

Finally, the third criterion emphasizes that human cognition, collaboration and performance is dependent upon context. It particularly looks at the representational context. That is, how the problem is represented influences the cognitive work needed to solve the problem(see Figure $\mathbf{2}$ and Zhang and Norman, 1994 Figure 2a representation of the tictac-toe game does not require any cognitive processing because the representation is perceptual and more efficient in terms of playing the game. However, an alternative or isomorphic representation of the tic-tac-toe game shown in Figure $2 b$ involves cognitive processing as the numbers in the circles need to be added up and thus is less efficient.

Problem solving is distributed across external and internal representations. Software systems based only on internal representations or models of a problem domain are likely to put a higher cognitive load on their users as against systems that are based on external or perceptual representations. Other contexts that need to be taken into account include social/organizational context and task can reach.

\section{Human-Centered System Development Framework}

In Figure 3 four components of the humancentered system development framework are shown. These are the activity-centered analysis, multi-layered multi-agent architecture component, transformation agent component, and multimedia interpretation component.

Human centered analysis must take account of varied social units that structure work and information, organizations and teams, communities and their distinctive social processes and practices. The underlying system dimension is social. The activity -centered analysis component models the social dimension and represents social quality component of the framework (Khosla et. al. 2003). The activity -centered analysis component is also based on human-centered criteria no. 2 outlined in section 2. The output of the activity-centered analysis component is the human-centered task model.

The goals for semantic quality are completeness and validity. Completeness is defined as the degree to which the software system represents each of the concepts in the stakeholder 痴 conceptualisations of

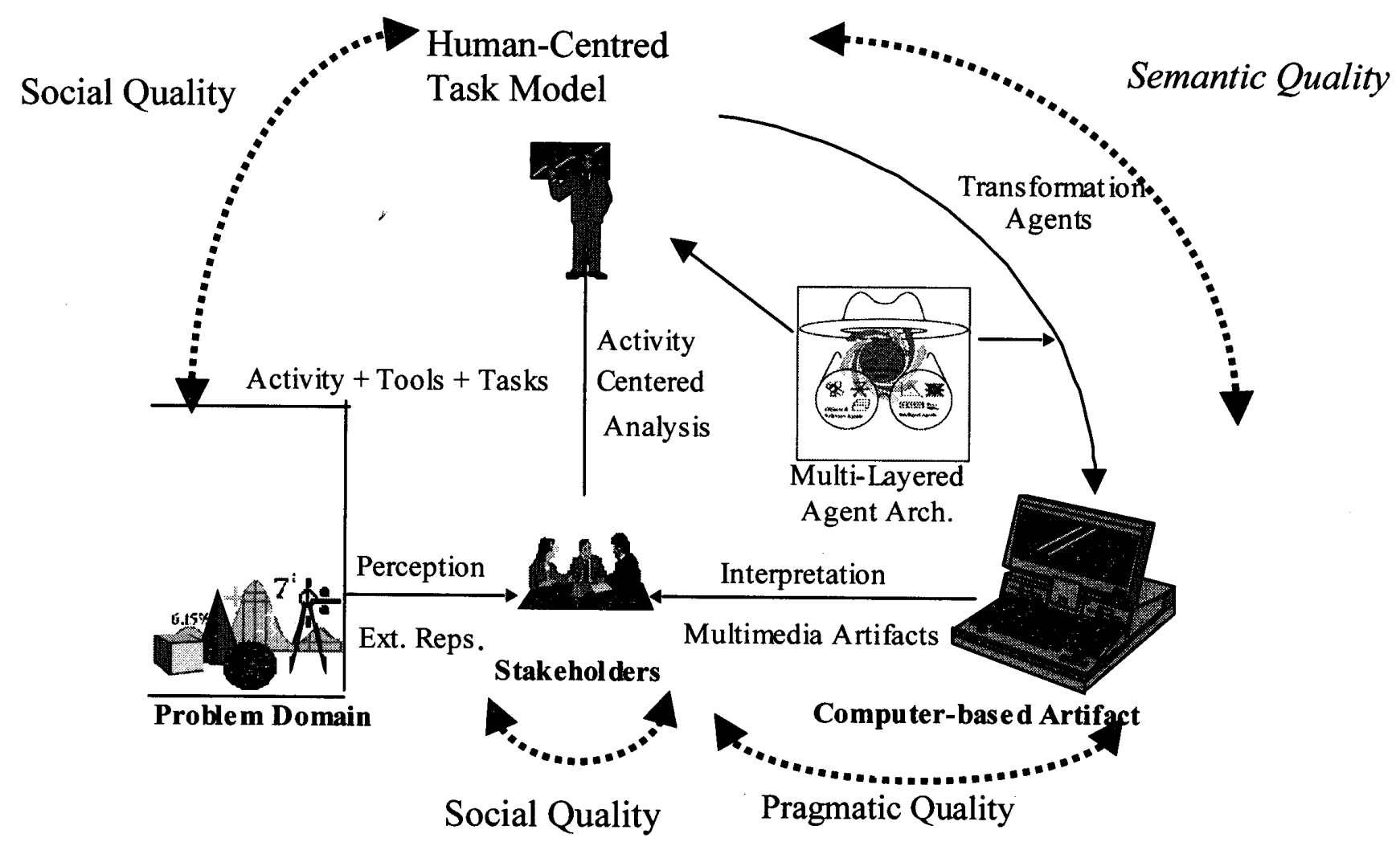

Figure 3: Human-Centred Systems Development Framework 
the domain of interest. Thus the question whose ideas get put into the design process is an important one for human centred Systems. As well, the question of whose problems are being solved is important. Systems which seek only to answer a very narrow technical or economic agenda or a set of theoretical technical points do not belong under the "human centred" rubric (Kling and Leigh, 1997). The distributed multi-layered multi-agent architecture component models the semantic quality dimension of the framework and is also based on human -centered criteria number 1 outlined in section 2 . The multi-agent architecture transforms the human -centered task model into human-centered computer based artefact using the transformation agent component. The transformation agent component provides agent based constructs for defining the five layers of the multi-agent architecture.

As with the architecture of buildings, the architecture of machines embody questions of liveability and usability. The underlying system dimension is the pragmatic dimension. The pragmatic dimension or pragmatic quality is modelled by the multimedia interaction component of the framework (Khosla et. al. 2000). This component is also based on human -centered criteria number 3 outlined in section 2 . In this paper we describe the distributed multi-layered multi-agent architecture component

\section{Distributed Multi-Agent Architecture for Intelligent Systems}

The multi-layered distributed agent architecture is shown in Figure 4. It is derived from integration of characteristics of intelligent artifacts like fuzzy logic, neural network and genetic algorithm, agents, objects and distributed operating system process model with problem solving ontology model (Khosla et. al. 2000 and 2003). It consists of five layers, namely, the object layer, which defines the data architecture or structural content of an application. The distributed processing agent layer helps to define the distributed processing and communication constructs used for receiving, depositing and processing data in a distributed environment. In the rest of this section we describe the problem solving agent, optimization agent and the technology or tool agent layers respectively. From the perspective of intelligent agent design we illustrate how these three layers facilitate human-centeredness at three

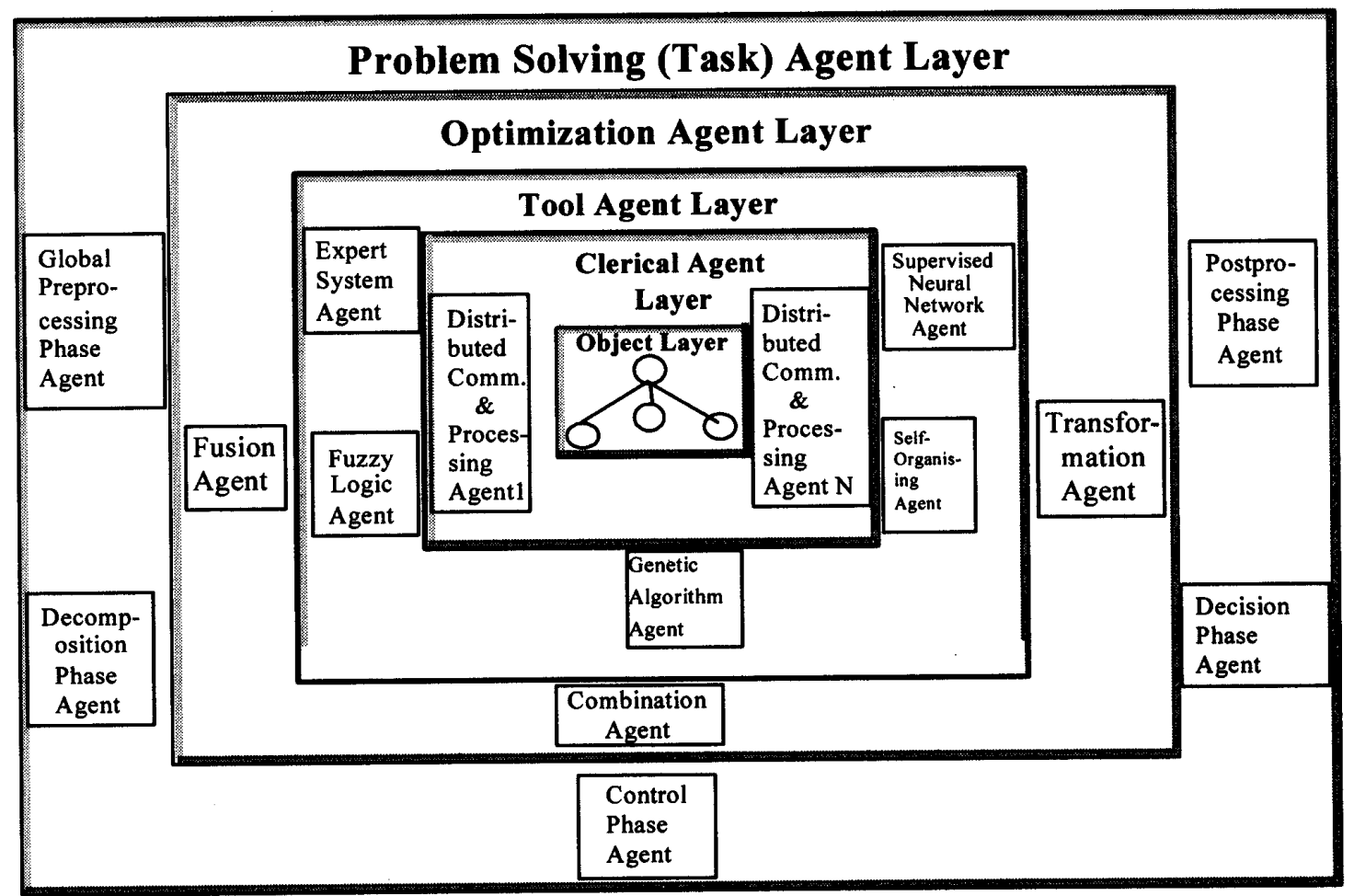

Figure 4: Multi-Layered Distributed Agent Ontology for Soft Computing Systems 
Table 1: Some Existing Problem Solving Ontologies

\begin{tabular}{|c|c|c|}
\hline Approach & Strengths & Some Limitations \\
\hline $\begin{array}{l}\text { Heuristic Classification (inference pattern) } \\
\text { Clancey } 1985\end{array}$ & $\begin{array}{l}\text { Good empirical generali- } \\
\text { zation }\end{array}$ & $\begin{array}{l}\text { No distinction between different classification } \\
\text { methods. (e.g. weighted evidence combination). } \\
\text { Not enough vocabulary. Pragmatic constraints } \\
\text { not considered. }\end{array}$ \\
\hline $\begin{array}{l}\text { Model Based Systems (part-whole, causal, } \\
\text { geometric, functional) (Steels 1985; Simmons } \\
\text { 1988) }\end{array}$ & $\begin{array}{l}\text { Principled domain } \\
\text { models, complete knowl- } \\
\text { edge }\end{array}$ & $\begin{array}{l}\text { Combinatorial explosion, assumes all observa- } \\
\text { tions are correct \& exact domain theory is } \\
\text { known. }\end{array}$ \\
\hline $\begin{array}{l}\text { Problem Solving Ontologies (between model } \\
\text { based and data based) based on Problem Solv- } \\
\text { ing Methods - (cover-and-differentiate, pro- } \\
\text { pose-test-refine, etc) (McDermott 1988; Fen- } \\
\text { sel 1996, 1997, 2000) }\end{array}$ & $\begin{array}{l}\text { Helps to determine type } \\
\text { of domain knowledge } \\
\text { required for problem } \\
\text { solving, eases the knowl- } \\
\text { edge acquisition bottle- } \\
\text { neck. }\end{array}$ & $\begin{array}{l}\text { When to stop the knowledge acquisition, when is } \\
\text { the system complete. Can lead to deep solution } \\
\text { hierarchies. Do not consider the role of external } \\
\text { representations or perceptual tasks } \\
\text { Primarily based on knowledge based systems. Do } \\
\text { not adequately consider pragmatic constraints } \\
\text { like human evaluation, response time, etc. }\end{array}$ \\
\hline $\begin{array}{l}\text { - Generic Task Based (classification, interpre- } \\
\text { tation, diagnosis \& construction/design) } \\
\text { (Chandrasekaran 1983, Chandrasekaran \& } \\
\text { Josephson 1997, Chandrasekaran \& Johnson } \\
\text { 1993, Steels 1990. }\end{array}$ & $\begin{array}{l}\text { Reuse, basis for interpret- } \\
\text { ing acquired data, can } \\
\text { build generic software } \\
\text { environments. }\end{array}$ & $\begin{array}{l}\text { Generic task categorization is not generic } \\
\text { enough because they (e.g. diagnosis) can be } \\
\text { accomplished using many different domain } \\
\text { models, and different methods (depending on } \\
\text { problem granularity), pragmatic constraints not } \\
\text { considered. Tasks only mediated by methods. }\end{array}$ \\
\hline $\begin{array}{l}\text { Generic Ontology KADS methodology } \\
\text { (domain layer, inference layer, task layer, } \\
\text { strategy layer) (Breuker and Weilinga 1989, } \\
\text { 91; Weilinga 1993.) }\end{array}$ & $\begin{array}{l}\text { Segregates knowledge } \\
\text { modeling into four } \\
\text { layers. }\end{array}$ & $\begin{array}{l}\text { System modeling done with low level primitives. } \\
\text { Suitable for knowledge based problems only. } \\
\text { Does not consider pragmatic constraints. }\end{array}$ \\
\hline
\end{tabular}

distinct levels.

\subsection{Problem Solving Ontology Layer}

The problem solving ontology (task) agent layer defines the constructs related to the problem solving agents namely, preprocessing, decomposition, control, decision and postprocessing. We discuss the definition of the problem solving agents in this section

This layer systematizes and organises practitioners/stakeholder/user's tasks in the domain under study. It also helps to model practitioner's problem solving approach and tasks in domains which are complex and data intensive. The research on problem solving ontologies or knowledge-use level architectures has largely been done in artificial intelligence. The research at the other end of the spectrum (e.g., radical connectionism, soft computing) is based more on understanding the nature of human or animal behavior rather than developing ontologies for dealing with complex real world problems on the web as well as in conventional applications. Table 1 shows some of the existing work done by various researchers in this area including their strengths and some limitations Firstly, especially with the advent of the internet and the web human (or user)-centeredness has become an important issue (NSF workshop on human-centered systems 1997) and Takagi (2001). Secondly, from a cognitive science viewpoint, distributed cognition (which among other aspects involves consideration of external and internal representations for task modelling) has emerged as a system modelling paradigm as compared to the traditional cognitive science approach based on internal representations only. Finally, as outlined in the last section there are a range of hard and soft computing technologies and their hybrid configurations which lend flexibility to the problem solver as against trying to force fit a particular technology or method on to a system design (as traditionally has been done). These three developments can be considered as pragmatic constraints. Most existing approaches do not meet one or more of the pragmatic constraints. Besides, from a soft computing perspective most existing architectures do not facilitate component based modelling at optimization, task and technology levels respectively. Some 
generic goals and tasks associated with each problem solving agent are shown in Table 2. This layer employs the services of the other 4 layers for accom. plishing various tasks.

\subsection{Optimization Layer}

The optimization agent layer defines constructs for fusion, combination and transformation technologies which are used for optimizing the quality of solution (e.g., accuracy). In this section we discuss the definition of the optimization agents.

The four most commonly used intelligent technologies are symbolic knowledge based systems (e.g. expert systems artificial neural networks, fuzzy systems and genetic algorithms). The computational and practical issues associated with intelligent technologies have led researchers to start hybridizing various technologies in order to overcome their limitations (Khosla et.al. 1997, 2000, 2003). However, the evolution of hybrid systems is not only an outcome of the practical problems encountered by these intelligent methodologies but is also an outcome of deliberative, fuzzy, reactive, self-organizing and evolutionary aspects of the human information processing system (Bezdek, 1994).

These hybrid configurations can be grouped into three classes, namely, fusion systems, transformation systems, combination systems (Khosla et.al. 2000; Khosla et. al. 1997 ; Chiaberage et. al., 1995) These classes along with individual technologies are shown in Figure 5 along two dimensions, namely, quality of solution and range of tasks.

In fusion systems, the representation and/or information processing features of in technology A are fused into the representation structure of another technology B. From a practical viewpoint, this augmentation can be seen as a way by which a technology addresses its weaknesses and exploits its existing strengths to solve a particular real-world problem.

Transformation systems are used to transform one form of representation into another. They are used to alleviate the knowledge acquisition problem. For example, neural nets are used for transforming numerical/continuous data into symbolic rules which can then be used by a symbolic knowledge
Table 2: Some Goals and Tasks of Problem Solving Agents

\begin{tabular}{|c|c|c|}
\hline Phase & Goal & Some Tasks \\
\hline Preprocessing & Improve data quality & $\begin{array}{l}\text { Noise Filtering } \\
\text { Input Condition- } \\
\text { ing }\end{array}$ \\
\hline Decomposition & $\begin{array}{l}\text { Restrict the context } \\
\text { of the input from the } \\
\text { environment at the } \\
\text { global level. By } \\
\text { defining a set of orth- } \\
\text { ogonal concepts } \\
\text { Reduce the complex- } \\
\text { ity and enhance over- } \\
\text { all reliability of the } \\
\text { computer-based arti- } \\
\text { fact }\end{array}$ & $\begin{array}{l}\text { Define orth- } \\
\text { ogonal concepts }\end{array}$ \\
\hline Control & $\begin{array}{l}\text { Determine decision } \\
\text { selection knowledge } \\
\text { constructs within an } \\
\text { orthogonal concept } \\
\text { for the problem } \\
\text { under study. }\end{array}$ & $\begin{array}{l}\text { Define decision } \\
\text { level concepts } \\
\text { with in each } \\
\text { orthogonal con- } \\
\text { cept as identified } \\
\text { by users } \\
\text { Determine Con- } \\
\text { flict Resolution } \\
\text { rules between } \\
\text { decision level } \\
\text { constructs }\end{array}$ \\
\hline Decision & $\begin{array}{l}\text { Provide decision } \\
\text { instance results in a } \\
\text { user defined decision } \\
\text { concept. }\end{array}$ & $\begin{array}{l}\text { Define decision } \\
\text { instances of } \\
\text { interest to the } \\
\text { user }\end{array}$ \\
\hline Post-processing & $\begin{array}{l}\text { Establish outcomes } \\
\text { as desired outcomes }\end{array}$ & $\begin{array}{l}\text { Concept valida- } \\
\text { tion } \\
\text { D e c i s i o n } \\
\text { instance result } \\
\text { validation }\end{array}$ \\
\hline
\end{tabular}

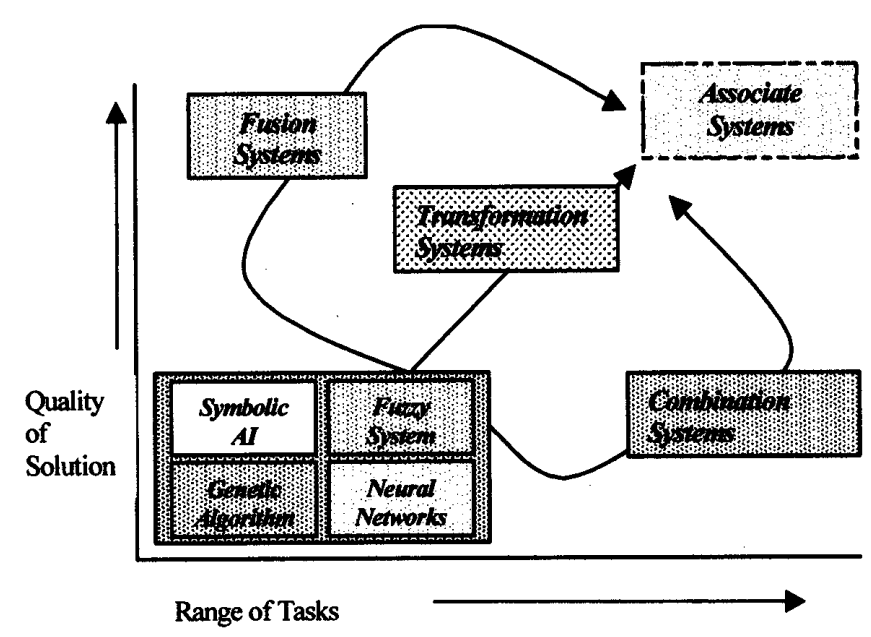

Figure 5: Technologies, Hybrid Configurations, Quality of Solution and Range of Tasks 
based system for further processing. Combination system involve explicit hybridization. Instead of fusion, they model the different levels of information processing and intelligence by using technologies that best model a particular level. These systems involve a modular arrangement of two or more technologies to solve real-world problems.

However, these hybrid architectures also suffer from some drawbacks. These drawbacks can be explained in terms of the quality of solution and range of tasks covered as shown in Figure 5. Fusion and transformation architectures on their own do not capture all aspects of human cognition related to problem solving. For example, fusion architectures result in conversion of explicit knowledge into implicit knowledge, and as a result lose on the declarative aspects of problem solving Thus, they are restricted in terms of the range of tasks covered by them. The transformation architectures with bottom-up strategy get into problems with increasing task complexity. Therefore the quality of solution suffers when there is heavy overlap between variables, where the rules are very complicated, the quality of data is poor, or data is noisy. The combination architectures cover a range of tasks because of their inherent flexibility in terms of selection of two or more technologies. However, because of lack of (or minimal) knowledge transfer among different modules the quality of solution suffers for the very reasons the fusion and transformation architectures are used. It is useful to associate these architectures in a manner so as to maximize the quality as well as range of tasks that can be covered. These class of systems are called associative systems as shown in Figure 5.

As may be apparent from Figure 5, associative systems consider various technologies and their hybrid configurations as technological primitives that are used to accomplish tasks. The selection of these technological primitives is contingent upon satisfaction of task constraints. In summary, it can seen from the discussion in this section that associative systems represent evolution from a technology-centered approach to a task-centered approach

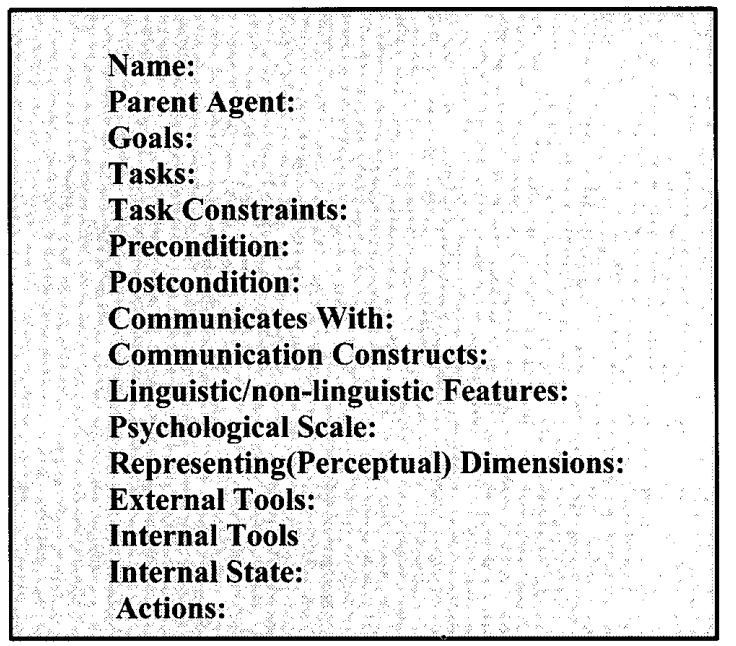

Figure 6: Generic Agent Definition

\subsection{Tool or Technology Layer}

The tool or technology agent layer defines the constructs for various intelligent and soft computing tools. Finally, The five layers facilitate a component based approach for agent based software design. The generic agent definition used for defining the agents in various layers is shown in Figure 6. The generic agent definition shown in Figure 6 includes goals which is a desire or desired outcome or state. Tasks: are goal directed processes in which people consciously or unconsciously engage. Task constraints: are pragmatic constraints imposed by the stakeholders and the environment for successful accomplishment of a task. The task constraints primarily determine the selection knowledge required for selecting a technological artifact Precondition helps us to define underlying assumptions for task accomplishment. Postcondition. defines the level of competence required from the technique or algorithm used for accomplishing the task. The communication constructs employed by the transformation agent. These communication constructs are based on human communicative acts like request, command, inform, broadcast, explain, warn and others. The linguistic and non-linguistic features represent the sensed data from the external environment as well as computed data by the agent. Representing Dimension is the physical or abstract dimension used to represent a feature. It can be seen as capturing the perceptual representation or category of a feature. These representing dimensions can be shape, color, distance, location, etc.. 


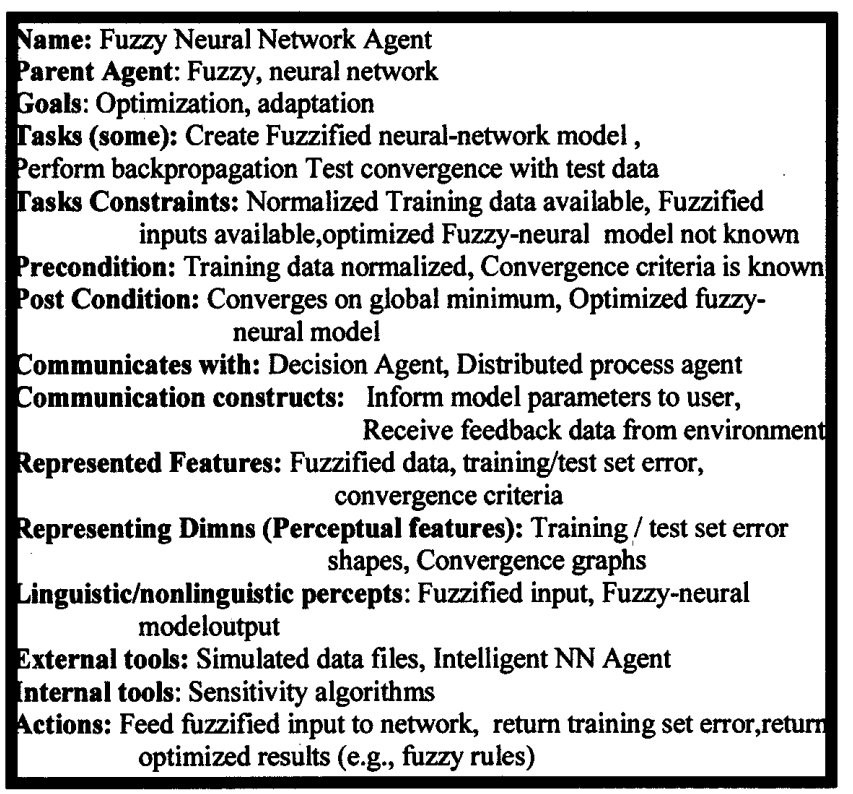

Figure 7: Fuzzy-Neural (Fusion) Agent

Psychological Scale is the abstract measurement property of the physical or abstract dimension of a represented feature. The parent agent construct identifies the generic agents in the four agent layers, whose constructs and services have been inherited by a particular application or domain based transformation agent. The communication with construct in Figure 6 identifies all the agents and objects that a transformation agent communicates with in the five layers. The external tools construct in Figure 6 refers to those computer-based or other tools that are external to the definition of an agent. On the other hand, internal tools are those tools that are defined internally by a transformation agent. The internal state construct refers to the beliefs of a transformation agent at a particular instant in time. Finally, the actions construct is used to define the sequence of actions for accomplishing various tasks. Figure 7 provides a sample fuzzy-neural network agent definitions at optimization level of the architecture.

\section{Human-Centered Modelling Using Multi-Layered Multi-Agent Architecture}

In this section we establish the human-centeredness of the multi-layered agent architecture from two perspectives. Firstly, the multi-layered agent ontology facilitates human involvement, evaluation and feedback at three levels, namely, task, optimization and technology respectively. The domain and technology independent constructs used in the problem solving agent layer are based on consistent problem solving structures employed by users in developing solutions to complex problems. These constructs facilitate a problem driven approach as required human-centered criteria number 1 . Further, the component based nature of the five problem solving agents allows them to be used in different problem solving contexts or sequences as shown in Figure 8. Figure 8 shows the decision paths taken by a medical practitioner for diagnosis and prescribing treatments to a patient. In one context the medical practitioner may not need the help of the computerized system for diagnosing a patient is disease and may employ the decision path from diagnosis to treatment. Whereas, in another context the medical practitioner may not know the diagnosis and thus seek the help of the computerized

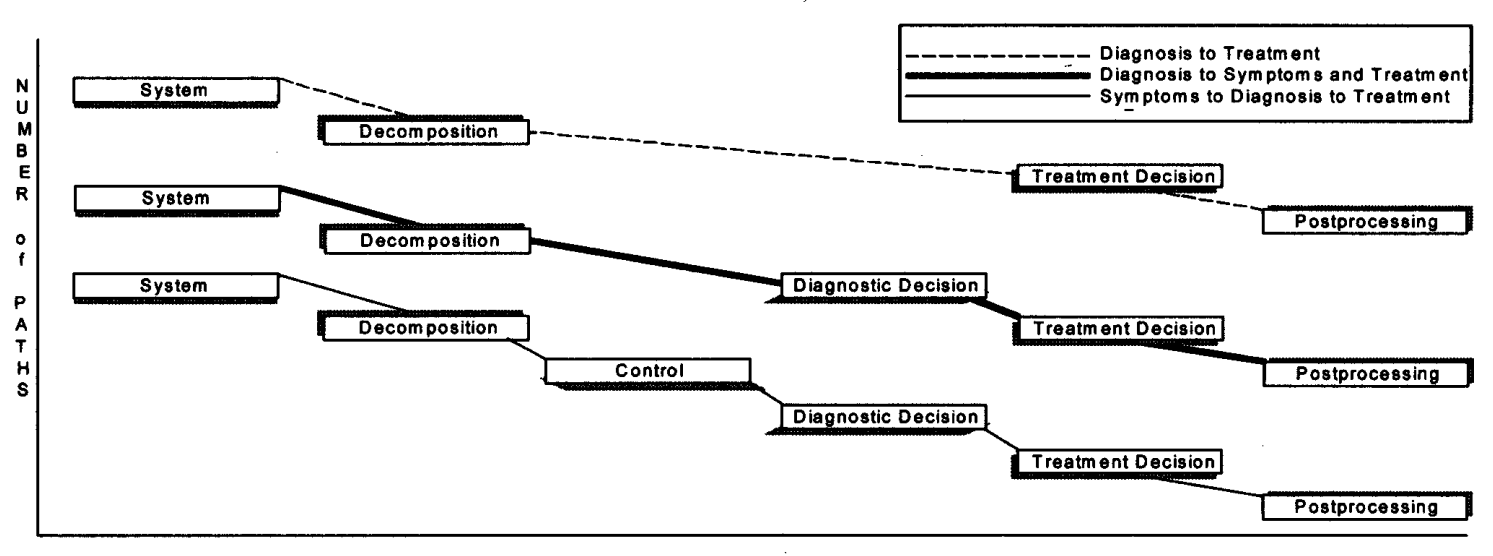

Decision Paths

Figure 8: Decision Paths Taken by a Medical Practitioner and Agent Sequence 
Table 3: Task Level mapping of content terms and attributes with those of the context-awar-e-commerce application platform

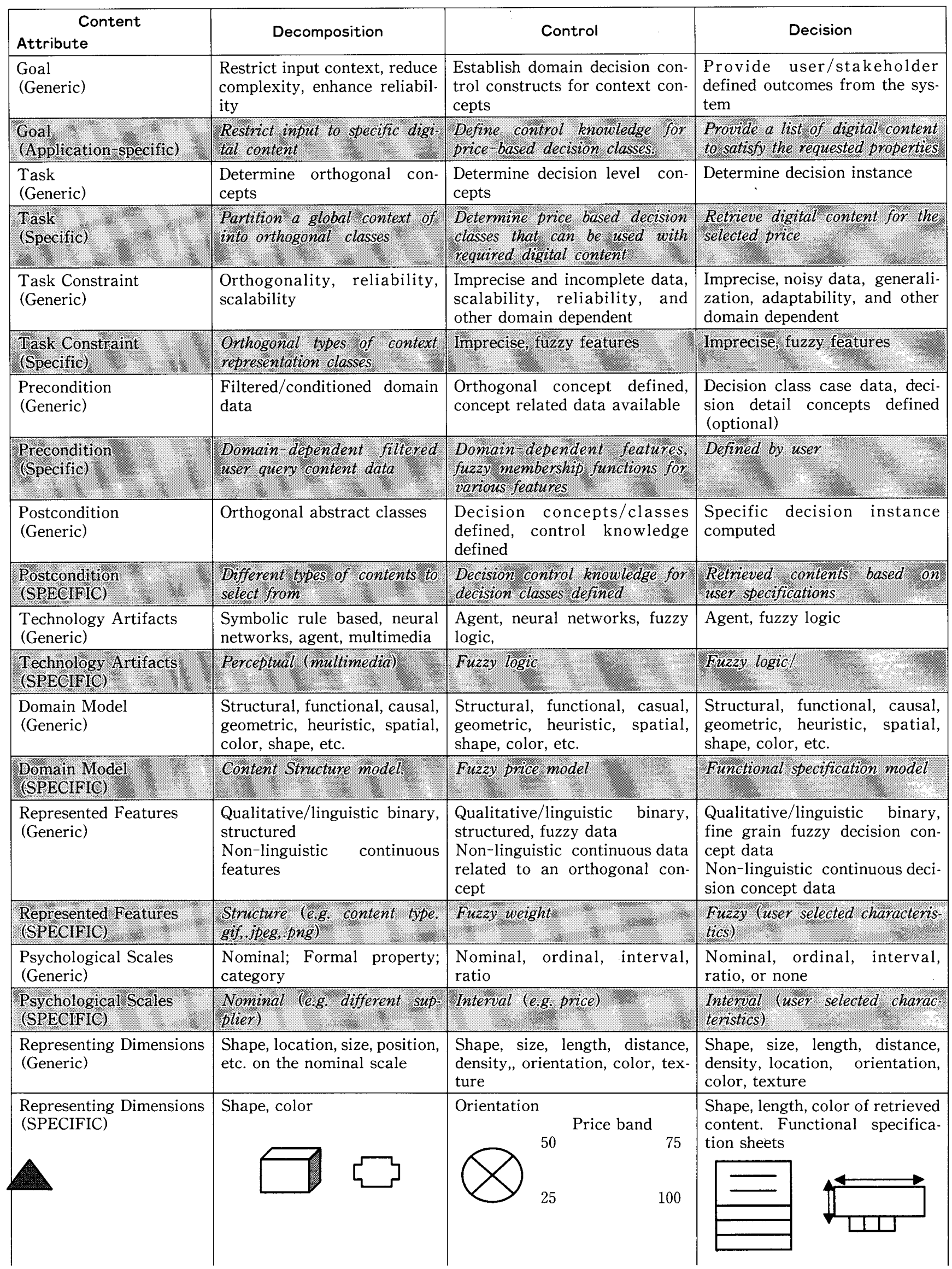




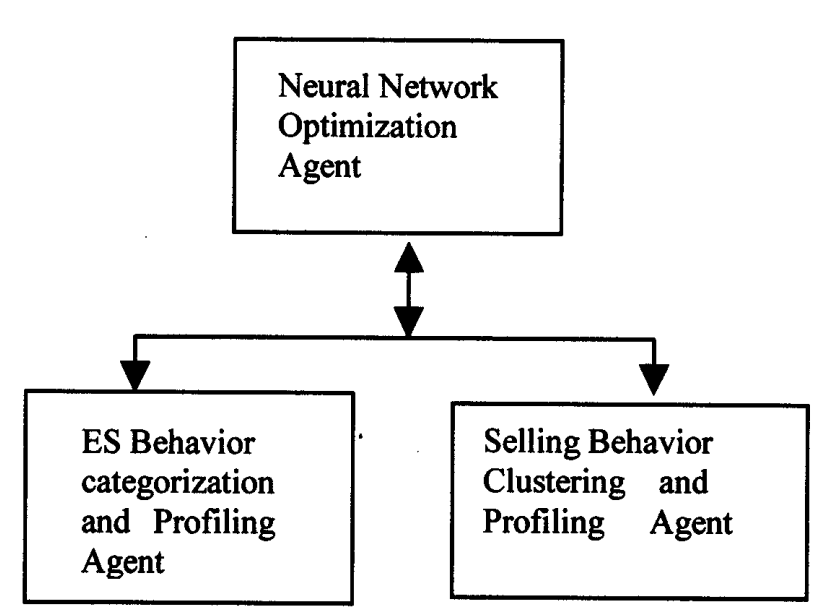

Figure 9: Optimization Level Modelling of e-SRS.

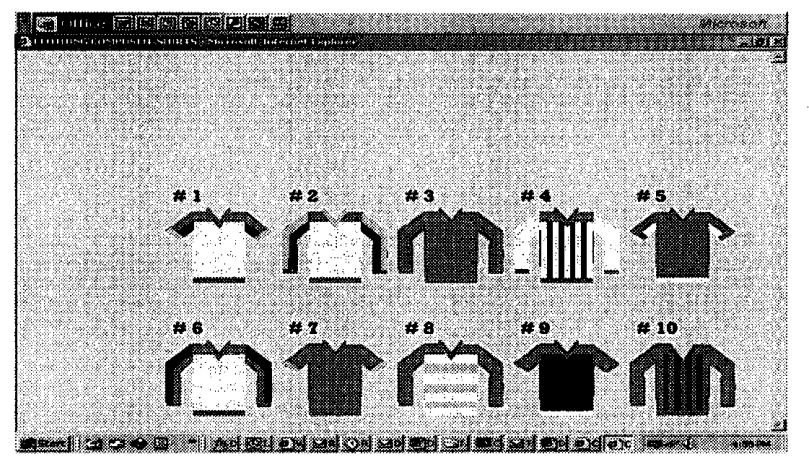

Figure 10: Interactive Shirt Design in a Web Based Missing Person Identification Application References

system from symptoms to diagnosis to treatment.

In Table 3 we show application of decomposition, control and decision problem solving agents to model a context based buying model of an internet user in an e-commerce application.

The optimization level allows an existing technology based solution to be optimized based on human feedback. For example, in a e-sales recruitment problem we use a neural network in combination with expert system agent and a clustering agent to optimize the performance of e-sales recruitment system as shown in Figure 9 The neural network agent is trained on positive and negative examples of acceptance and rejection by a recruitment manager of a sales candidate's behavior category as predicted by ES agent and clustering agent respectively.

At the technology level human involvement is encouraged by asking user to define the search parameters or fitness function to be used by a soft computing agent like GA to create the correct combination of shirt design as shown in Figure 10. .Further, the generic tasks of the five problem solving agents have been grounded in human-centered criteria outlined in the 1997 NSF workshop on human-centered systems. These criteria state that a) human-centered research and design is problem driven rather than logic theory or any particular technology; b) human-centered research and design focuses on practitioner's goals and tasks rather than system developer's goals and tasks, and c) humancentered research and design is context bound. The context criterion relates to social/organizational context and representational context (where people use perceptual as well as internal representations to solve problems). The generic tasks employed by the five problem solving agents are based on consistent problem solving structures used by practitioners solving complex problems in engineering, process control, image processing, management and other areas. The perceptual dimension (e.g., color, shape, texture, etc.) and psychological scale (e.g., nominal, ordinal, equal interval and ration (Steven, 1957) constructs shown in Figure 6 as part of the generic agent definition model the representational context. Further, the social and organization context (not described in this paper) has also been modelled (khosla, 2003) Additionally, the five layers of the agent ontology lead to component based distributed (collaboration and competition) soft computing system design. The ontology also provides flexibility of technologies, learning and adaptation, different forms of knowledge (fuzzy, rule based and distributed pattern based) to be used for modelling. component based software design.

\section{Conclusion}

In this paper we have outlined a multi-layered distributed multi-agent for developing soft computing applications. The multi-layered architecture provides human-centered modeling support at three levels, namely, task level, optimization level and technology level. Human-centered modeling support is provided in terms developing a humancentered problem solving model, using human feed- 
back for optimizing a technology based solution and using human evaluation to determine the objective function for a soft computing search technique. Application examples are used to illustrate modelling at three levels. The architecture has been applied in a range of areas including web mining, image processing, e-commerce, alarm processing, medical diagnosis and others..

\section{References}

[1] Bezdek, J.C. (1981). Pattern Recognition with Fuzzy Objective Function Algorithms: Advanced Applications in Pattern Recognition, Plenum Press, USA

[2] Bezdek, J.C. (1991). What is Computational Intelligence? Computational Intelligence: Imitating Life, Eds. Robert Marks-II et al., IEEE Press, New York.

[ 3 ] Breuker, J.A. and Weilinga, B.J., (1989) Model Driven Knowledge Acquisition in B. Guida \& G. Tasso eds. Topics in the Design of Expert Systems, Springer-Verlag,pp 23980.

[ 4 ] Breuker, J.A. and Weilinga, B.J., (1991) Intelligent Multimedia, Edited by Mark Maybury, AAAI Press, Menlo Park, CA.

[5] Buzzotte, V. R., Lefton, R. E., Sherberg, M. (1981). Effective Selling through Psychology, Psychological Associates, New York.

[6] Chandrasekaran B. and Josephson, J.R., (1997). Ontology of Tasks and Methods, AAAI 97 Spring Symposium on Ontological Engineering, March 24-26, Stanford University, CA California, USA.

[ 7 ] Chandrasekaran, B. (1983). Towards Taxonomy of Problem Solving Types, AI Magazine Vol 4 No. 1, Winter/Spring pp 9-17.

[ 8 ] Chandrasekaran, B., and Johnson, T.R. (1993). Generic Tasks and ask Structues: History Critique and New Directions, Second Generation Expert Systems

[ 9 ] Clancey, W.J., (1985). Heuristic classification, Artificial Intelligence, 27, 3 (1985), 289-350.

[10] Chiaberage, M., Bene. G.D., Pascoli, S.D., Lazzerini, B., and Maggiore, A. (1995). Mixing fuzzy, neural \& genetic algorithms in integrated design environment for intelligent controllers, 1995 IEEE Int Conf on SMC,. Vol. 4, pp.
2988-93.

[11] Fensel, D. and Groenboom, R., (1996). MLPM: Defining a Semantics and Axiomatization for Specifying the Reasoning Process of Knowledge-based Systems,.ECAI, pp 423-427

[12] Fensel, D (1997). The Tower-of-Adapter Method for Developing and Reusing ProblemSolving Methods, EKAW, pp. 97-112

[13] Fensel, D. (2000). Problem-Solving Methods: Understanding, Development, Description, and Reuse, in Lecture Notes on Artificial Intelligence, no 1791, Springer-Verlag, Berlin.

[14] Goldberg, D.E. (1989). Genetic Algorithms in Search, Optimization and Machine Learning, Addison-Wesley, Reading, MA.

[15] Khosla, R., and Dillon, T. (1997a). Learning Knowledge and Strategy of a Generic NeuroExpert System Architecture in Alarm Processing, IEEE Transactions on Power Systems, Vol. 12, No. 12, pp. 1610-18.

[16] Khosla, R., and Dillon T. (1997b). Engineering Intelligent Hybrid Multi-Agent Systems, Kluwer Academic Publishers, MA, USA August 1997.

[17] Khosla, R., Sethi, I. and Damiani, E. (2000). Intelligent Multimedia Multi-Agent Systems A Human-Centered Approach, Kluwer Academic Publishers, MA, USA.

[18] Khosla, R. Damiani, E., and Grosky, W. (2003). Human-Centered E-Business, Kluwer Academic Publishers, MA, USA.

[19] Kling, R., and Leigh, S. (1997), http://www. slis. indiana. edu/CSI/wp97-04.html.

[20] McDermott, J. (1988). Preliminary steps toward a taxonomy of problem solving methods. In Automated Knowledge Acquisition for Expert Systems, S. Marcus, Ed., Kluwer Academic, pp. 225-256.

[21] Simmons, R. (1988). Generate, Test, and Debug: A Paradigm for Solving Interpretation and Planning Problems. Ph.D diss., AI Lab, Massachusetts Institute of Technology.

[22] Steels, L. (1990). Components of Expertise, AI Magazine, 11, 28-49., 11, 28-49

[23] Steven, S.S. (1957). On the Psychological Law, Psychological Review, 64 (3), 153-181

[24] Takagi, H.K. (2001). Interactive Evolutionary 
Computation: Fusion of the Capabilities of EC Optimization and Human Evaluation, Proceedings of the IEEE, vol. 89, No. 9, September

[25] Zhang, J., Norman, D. A. (1994). Distributed Cognitive Tasks, Cognitive Science, pp. 84-120

(Received July 7, 2003)

[Contact Address]

School of Business

La Trobe University

Melbourne, Victoria 3086

Australia

Tel: (613) 94793064

Fax: (613) 94795971

E-Mail:R.Khosla@ latrobe.edu.au

\section{Information about Author}

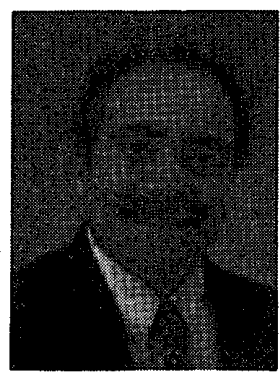

Rajiv Khosla holds degrees in Engineering (B.E. Electrical Engg), Management (M. Tech management and Systems), and Computer Science (M. Sc. and Ph.D.). He has been a visiting professor in Department of Computer Science, Wayne State University, Michigan, USA. in 1999. He has authored 3 research books (one a world first) and contributed to 7 others in soft computing agents, intelligent systems, multi-agent systems, human-centered systems, multimedia technologies and E-Business He has published over 100 papers in most areas in computer science and information systems. He is Associate Editor of International Journal of Pattern Recognition, Editorial Board member of International Journal on Knowledge Based Intelligent Engineering Systems, Review Board Member - International Journal of Applied Intelligence and Director of Business systems and Knowledge Modelling Laboratory

He has been actively involved with the industry as a project leader and innovator in more than a dozen industry based research projects in the last five years in information technology area. A number of software products have also been commercialized. He has been a consultant to industry in the private and public sector. He is also the Director of Business Systems and Knowledge Modelling Laboratory. 